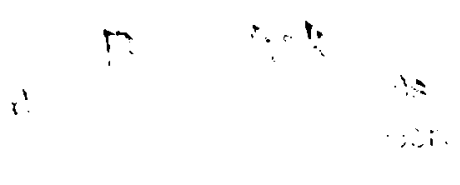

\title{
VII. Ein Beitrag zur Theorie von Barlow und Pope.
}

\author{
Von \\ F. M. Jaeger in Zaandam (Holland).
}

(Mit 1 Textfigur.)

\$1. Die geistreiche Theorie der Herren W. Barlow und W. J. Pope ${ }^{1}$, welche den Zusammenhang zwischen chemischer Constitution der festen Kürper und ihren Krystallformen abzuleiten versucht auf Grund der sterischen Verhältnisse der jene zusammensetzenden Atome und Radicale ${ }^{2}$, indem sie die Volumina der Wirkungssphären der einzelnen Atome in jeder chemischen Verbindung proportional deren Valenzzahl setzt, sagt auch bestimmte Formanalogien voraus für den Fall, daB die einander vertretenden Radicale sich um eine paare Zahl von Valenzeinheiten unterscheiden. Wenn z. B. ein Stickstoffatom vom Volum $=3$ ein Kohlenstoffatom vom Volum $=4$ substituiert, so kann die ursprüngliche homogene Atomanordnung nur erhalten oder fast erhalten bleiben, wenn aus der Umgebung des substituierten Atoms gleichzeitig noch ein einwertiges Atom austritt. Als Beispiele für diese Folgerung ihrer Valenzinterpretation führen die beiden Autoren u. a. folgende Tatsachen an: Die bekannte Formanalogie zwischen Calcit und Natronsalpeter einerseits und zwischen $\Lambda$ ragonit und Kalisalpeter andererseits ${ }^{3}$; die von Zirngiebl $\left.{ }^{4}\right)$ gefundene Isomorphie zwischen den Salzen von Methandisulfon-

1) W. Barlow und W. J. Pope, Trans. of Chem. Soc. 1906, 89, 1675-1744 und 91, 1150. Vergl. die späteren Ausführungen von G. Le Bas, über die Valenzvolumina der homologen Paraffine und deren Verbrennungswärme, Journ. Chem. Soc. 1907, 91, 112 und Proceed. 1907. Auch: Report of the British Association Leicester 1907.

2) Vergl. F. M. Jaeger, diese Zeitschr. 38, 596-639; ebenda 40, 146. Dissertalion Leiden 1903, S. 190-195.

3) Pope und Barlow, l. c. S. 1732 .

4) Zirngiebl, diese Zeitschr. 1902, 36, 117. 
sïure $\mathrm{CII}_{2}\left(\mathrm{SO}_{3} \mathrm{II}\right)_{2}$ und der Iminosulfonsäure $\left.\mathrm{NH}_{2} \mathrm{SO}_{3} \mathrm{H}\right)_{2}$; die Formverwandtschaft zwischen dem von Benedicks ${ }^{1}$ ) gemessenen Para-Nitrobenzolsulfonsäurechlorid und dem correspondierenden Amid; diejenige zwischen der Orthosulfobenzoësäure, deren Chloride (labil und stabil) und dem Saccharina) $0-\mathrm{C}_{6} \mathrm{H}_{4}<_{\mathrm{SO}_{2}}^{\mathrm{CO}}>\mathrm{NH}$; zwischen Kaliumchromat und Chromsäureanhydrid ${ }^{3}$ ). Ich konnte noch hinzufügen: zwischen Jodoform und Phosphortrijodid, zwischen dem Benzylidenanilin und dem Stilben und zwischen Dibenzyl und Benzylanilin ${ }^{4}$ ) usw.

\$ 2. Im folgenden kann ich einen neuen bemerkenswerten Beitrag zu diesen theoretischen Schlußfolgerungen vorführen. Herr Pope schrieb mir, dal er eine krystallographische Formanalogie erwartete zwischen dem Saccharin und dem Phtalimid und auch zwischen ihnen und der $\Lambda \mathbf{n}$ thranilsäure:

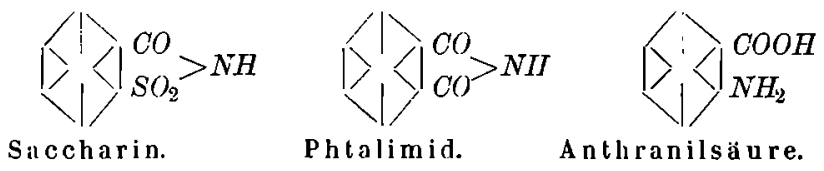

Es sollte nïmlich die Substitution von $-\mathrm{SO}_{2}-$ (Volum 6) durch - $\mathrm{CO}-$ (Volum 6) keine merkliche Abänderung der krystallographischen Molekularanordnung zur Folge haben künnen, während in dem Falle der beiden letzten Substanzen jedenfalls größere Differenzen erwartet werden konnten.

Nach vielen vergeblichen Versuchen gelang es mir, das Phtalimid in schön krystallisierter Form zu erhalten. Die Anthranilsäure ist schon vor lïngerer Zeit von II a ushofer ${ }^{5}$ ) gemessen worden. Ich fand dieselben Parameter und Winkelwerte, so daß ich daher nur auf die betreffende Literaturstelle zu verweisen brauche.

Die Anthranilsäure (Ortho-Amidobenzoësäure) ist rhombischbipyramidal mit $a: b: c=0,3959: 1: 0,8601$. Nimmt man hierin $\frac{3}{2} c$, d. i. durch dieselbe Zahl multipliciert wie beim Saccharin $\left.{ }^{6}\right)$ und beim Phtalimid (s. daselbst), so wird $a: b: c=0,5959: 1: 1,2901$ und, weil das Valenzvolum hier $=42$ ist, berechnen sich die Äquivalenzparameter zu $x: y: \approx=2,2612: 3,7945: 4,8952$; diese Werte sind jenen der Ortho-

1) Benedicks, diese Zeitschr. 1903, 37, 285.

2) Pope und Barlow, 1. c. S. 1734.

3) Ebenda S. 1736.

4) F. M. Jaeger, Versl. kon. Akad. v. Wetensch. Amsterdam, englische Ausg. 1906, P. 460.

i) Ilaushofer, diese Zeitschr. 1879, 1, 50:.

6) Pope und Barlow, l. c. S. 1734. 
Sulfobenzö̈säure ${ }^{1}$, des stabilen und labilen Ortho-Sulfobenzoësäurechlorids ${ }^{1}$ ) und des Saccharins ${ }^{1}$ ) ganz analog, wie auch denen des Phtalimids, wie weiter unten bewiesen wird.

Das Phtalimid, Schmelzpunkt $238^{\circ} \mathrm{C}$., ist monoklin-prismatisch und zeigt bei allem Formenunterschied geometrisch die gröBte Analogie mit dem Saccharin, wie untenstehende Vergleichung zeigt:

Saccharin: Monoklin-prismatisch. $a: b: e=1,3560$ : 1:0,4298;

$\beta=94^{\circ} 3^{\prime}$. Vollkommen spaltbar nach $\{100\}$.

Phtalimid: Monoklin-prismatisch. $a: b: c=1,4913: 1: 0,4967$;

$\beta=91^{0} 18 \frac{2}{3}$. Vollkommen spaltbar nach $\{100\}$.

Berechnet man für letztere Verbindung die Äquivalenzparameter, wie für das Saccharin geschehen ist, so werden dieselben:

$$
x: y: \approx=2,4623: 3,3022^{\circ}: 4,919 \% \text {. }
$$

Wir haben jetzt also:

$$
\begin{array}{ll}
\text { o-Sulfobenzoësäure: } & x: y: z=2,5708: 3,8496: 4,6481 . \\
\text { Labiles Chlorid: } & x: y: z=2,5727: 3,6773: 4,4377 . \\
\text { Stabiles Chlorid: } & x: y: z==2,4770: 3,7025: 4,5800 . \\
\text { Saccharin: } & x: y: z=2,5183: 3,6857: 4,7521 . \\
\text { Phtalimid: } & x: y: \approx=2,4623: 3,3022: 4,9197 .
\end{array}
$$

Iliermit ist die von der neuen Theorie vorausgesagte Tatsache experimentell bewiesen.

\section{Phtalimid.}

Structur:

$$
\int_{C O}^{C O}>N H .
$$

Schmelzpunkt $238^{\circ}$ C.

Aus einem Gemisch von Äther und Äthylacetat kann man die Verbindung bei schr langsamer Verdampfung erhalten in Form kleiner, außerordentlich stark lichtbrechender, farbloser und sehr glänzender Nädelchen, welche tadellos gebaut sind und ziemlich constante Winkelwerte aufweisen.

Monoklin-prismatisch.

$$
a: b: c=1,4913: 1: 0,4967 ; \beta=91018 \%{ }^{\prime} .
$$

Beobachtete Formen: $a\{100\}$ groß und stark glänzend; $m\{110\}$ etwas schmäler, aber gute Reflexe liefernd; $q\{011\}$

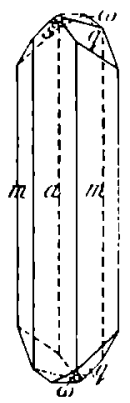
groß, ideal reflectierend; $\omega\{(122\}$ etwas schmäler und lichtschwächere Reflexe liefernd; $r\{T 02\}$ klein, aber gut glänzend; $s\{102\}$ klein,

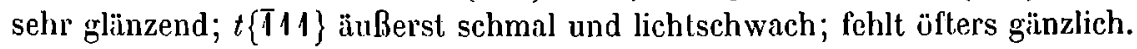

1) Pope und Barlow, 1. c. S. 173\%. 


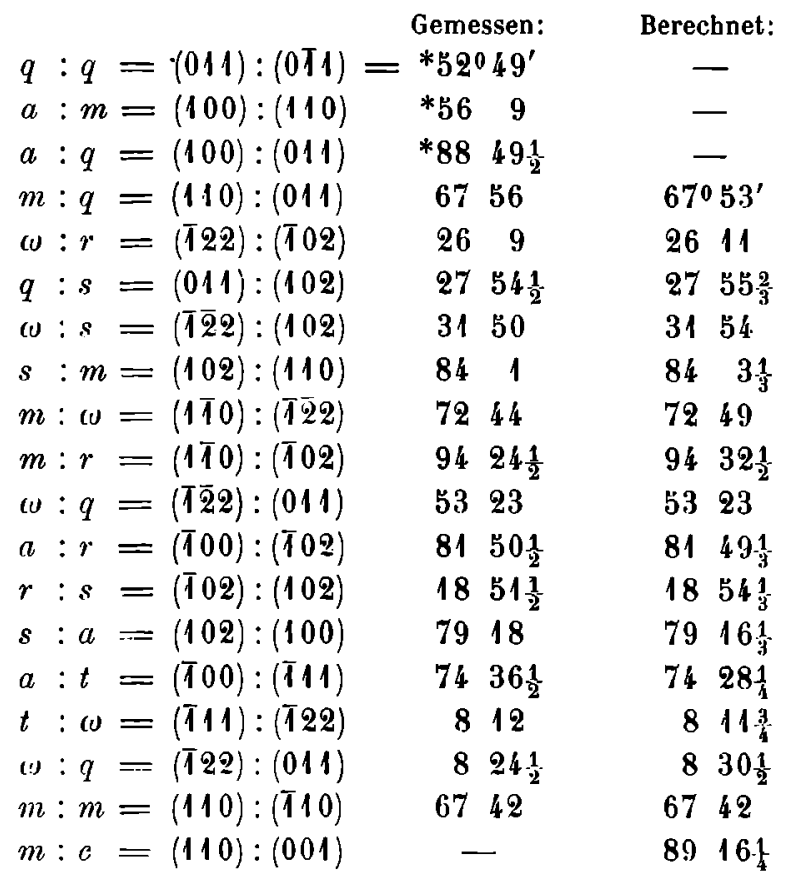

Sehr vollkommen spaltbar nach $\{100\}$ mit glänzender Spaltungsfläche. Die Übereinstimmung zwischen Messung und Rechnung ist vorzüglich, was als Beleg gelten kann für die geometrische Genauigkeit des Krystallbaues. - Auslöschung auf $\{100\}$ parallel zur $c-\Lambda x e$, auf $\{110\}$ circa $19^{\circ} \mathrm{da}-$ gegen geneigt. 